\title{
Real-time TaqMan PCR for rapid detection and typing of genes encoding CTX-M extended- spectrum $\beta$-lactamases
}

\begin{abstract}
Correspondence
Christopher I. Birkett

christopher.birkett@

addenbrookes.nhs.uk
\end{abstract}

Received 22 August 2006

Accepted 22 September 2006

\author{
Christopher I. Birkett, ${ }^{1}$ Hugo A. Ludlam, ${ }^{1}$ Neil Woodford, ${ }^{2}$ \\ Derek F. J. Brown, ${ }^{1}$ Nicholas M. Brown, ${ }^{1}$ Mark T. M. Roberts, ${ }^{1}$ \\ Nicola Milner ${ }^{1}$ and Martin D. Curran ${ }^{1}$ \\ ${ }^{1}$ Health Protection Agency, Clinical Microbiology and Public Health Laboratory, Addenbrooke's \\ Hospital, Cambridge, UK \\ ${ }^{2}$ Antibiotic Resistance Monitoring and Reference Laboratory, Centre for Infections, Health \\ Protection Agency, London, UK
}

The prevalence of CTX-M-producing members of the Enterobacteriaceae is increasing worldwide. A novel, multiplex, real-time TaqMan PCR assay to detect and type b/a $\mathrm{CTX}_{\mathrm{M}} \mathrm{M}$ genes is described which is an improvement on previously described techniques with respect to reduced assay time, elimination of the need for protracted post-PCR processing and the convenience of a single reaction vessel. Based on $\beta$-lactam antibiogram and MIC data, 478 of 1279 Enterobacteriaceae isolates from clinical blood and urine culture specimens were selected and tested for extended-spectrum $\beta$-lactamase (ESBL) production using phenotypic methods. The new TaqMan assay detected and typed bla $\mathrm{CTX}_{\mathrm{M}}$ genes in 21 of $28 \mathrm{ESBL}$-producing isolates.

\section{INTRODUCTION}

Delayed treatment of infections caused by extendedspectrum $\beta$-lactamase (ESBL)-producing organisms is associated with increased mortality (Paterson et al., 2001, 2004). Originally described in 1989 (Bauernfeind et al., 1990), CTX-M-type ESBLs may now be the most common ESBL type worldwide (Paterson \& Bonomo, 2005). More than 50 different CTX-M enzymes have been described and can be assigned to one of five phylogenetic groups, CTX-M1, CTX-M-2, CTX-M-8, CTX-M-9 or CTX-M-25, based on their amino acid sequence (Bonnet, 2004). Molecular-based detection and typing of ESBL producers offers the potential for faster diagnosis and earlier epidemiological information for outbreak control than current practices employing locally based ESBL detection by culture methods combined with reference-laboratory-based typing by multiple PCR assays. We describe a multiplex, real-time TaqMan PCR method to detect and characterize CTX-M genotypes that is an improvement on previously described techniques.

\section{METHODS}

Blood culture isolates. Over the 2-year period from January 2002 to December 2003, 322 members of the Enterobacteriaceae were isolated from blood cultures received by the Microbiology laboratory at Addenbrooke's Hospital, Cambridge, UK. All Enterobacteriaceae isolates reported resistant to cefotaxime and/or ceftazidime when

Abbreviation: ESBL, extended-spectrum $\beta$-lactamase. originally isolated, or with reduced susceptibility to either of these agents in MIC tests on recovery from storage at $-70^{\circ} \mathrm{C}$, were identified to species level using API 20E kits (bioMérieux). ESBL production was tested by a combination disc method (Carter et al., 2000), with all available cephalosporin-clavulanate combinations (cefotaxime, ceftazidime, cefpirome and cefpodoxime; Oxoid).

Urine culture isolates. In a separate study, 957 isolates of the Enterobacteriaceae from consecutive urine samples received by the Microbiology laboratory between April and August 2004 were examined. All isolates resistant to ampicillin, amoxicillin-clavulanate or cefalexin by routine disc diffusion tests were tested for ESBL production by the combination disc method, as described above.

Design of the consensus primer pair and four Taqman probes. Reference $b l a_{\mathrm{CTX}-\mathrm{M}}$ sequences representing each of the five CTX-M phylogenetic groups were assembled from the GenBank database (http://www.ncbi.nlm.nih.gov/GenBank). Accession numbers used were: X92506 $\left(b l a_{\mathrm{CTX}-\mathrm{M}-1}\right)$, X92507 $\left(b l a_{\mathrm{CTX}-\mathrm{M}-2}\right)$, AF550415 $\left(b l a_{\mathrm{CTX}-\mathrm{M}-3}\right), \quad$ AJ005044 (bla $\left.a_{\mathrm{CTX}-\mathrm{M}-5}\right), \quad$ AJ005045 (bla $\left.a_{\mathrm{CTX}-\mathrm{M}-7}\right)$,

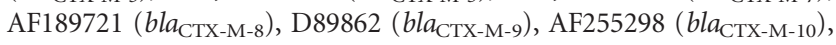
AJ310929 (bla $\left.a_{\text {CTX-M-11 }}\right), \quad$ AY571969 $\left(b l a_{\text {CTX-M-12 }}\right), \quad$ AF252622 $\left(b l a_{\text {CTX-M-14 }}\right)$, AY463958 (bla $\left.a_{\text {CTX-M-15 }}\right)$, AF325133 (bla $\left.a_{\text {СTХ-M-18 }}\right)$, AF518567 (bla $\left.a_{\text {CтХ-M-25 }}\right)$, AY157676 (bla $\left.a_{\text {CTХ-M-26 }}\right)$ and AF501233 $\left(b l a_{\mathrm{KLUG}-1}\right)$. The sequences were aligned using computer software [MegAlign (ClustalV method), Lasergene (version 5); http:// www.DNAstar.com]. Conserved sites were identified and a consensus primer pair was designed (Table 1; Metabion). Four TaqMan probes were designed around group-specific motifs: three of the probes were designed specifically to detect genes encoding CTX-M-1, CTXM-2 and CTX-M-9 group enzymes (Metabion); the fourth probe was designed to detect $b l a_{\mathrm{CTX}-\mathrm{M}}$ genotypes not belonging to the CTX-M-1 group and contained a minor groove binder (MGB) moiety to increase the melting temperature $\left(T_{\mathrm{m}}\right)$ and specificity 
Table 1. Sequences of the consensus primer pair and the four TaqMan probes for CTX-M genes

\begin{tabular}{|ll|}
\hline Primer/probe description & \multicolumn{1}{c|}{ Nucleotide sequence $^{\star}$} \\
\hline Forward CTX-M consensus primer & 5' $^{\prime}$-ATG TGC AGY ACC AGT AAR GTK ATG GC-3' \\
Reverse CTX-M consensus primer & 5'-ATC ACK CGG RTC GCC XGG RAT-3' \\
CTX-M-1 group probe & $5^{\prime}$-Joe-CCC GAC AGC TGG GAG ACG AAA CGT-Tamra-3' \\
CTX-M-2 group probe & $5^{\prime}$ - Cy5-CAG GTG CTT ATC GCT CTC GCT CTG TT-BHQ-2-3' \\
CTX-M-9 group probe & 5'-Rox-CTG GAT CGC ACT GAA CCT ACG CTG A-BHQ-2-3' \\
CTX-M probe for all groups except CTX-M-1 & 5'-6-Fam-CGA CAA TAC NGC CAT GAA-MGB-NFQ-3'
\end{tabular}

${ }^{\star} \mathrm{K}=\mathrm{G}$ or $\mathrm{T} ; \mathrm{N}=\mathrm{T}$ or $\mathrm{C} ; \mathrm{R}=\mathrm{A}$ or $\mathrm{G} ; \mathrm{Y}=\mathrm{C}$ or $\mathrm{T} ; \mathrm{X}$ (inosine) $=\mathrm{A}, \mathrm{C}, \mathrm{G}$ or $\mathrm{T}$.

(Applied Biosystems UK). Probe reporter dyes (at the $5^{\prime}$ position) were either Fam, Cy5, Rox or Joe and quencher dyes (at the 3' position) were either Tamra, BHQ-2 or NFQ (Table 1).

Positive control strains. Isolates producing CTX-M-9 (Enterobacter sp., ref. E395), CTX-M-14 (Escherichia coli, ref. 42032), CTX-M-15 (E. coli, ref. 0516) and CTX-M-2 (E. coli, ref. H042680216) were obtained from the Antibiotic Resistance Monitoring Reference Laboratory (ARMRL, Centre for Infections, Health Protection Agency, London, UK) for use as positive PCR controls.
DNA extraction and the PCR assay. Two c.f.u. of pure organism were emulsified in $10 \mathrm{ml}$ sterile Luria-Bertani (LB) broth and incubated overnight at $37^{\circ} \mathrm{C}$. A pellet was obtained by centrifugation and total DNA was extracted using a modification of the method described by Narayanan et al. (2001). A final DNA concentration of 2-20 ng $\mu \mathrm{l}^{-1}$ was used in the PCR assay. Once optimal reaction conditions had been determined empirically using the Rotor-Gene 3000 apparatus (Corbett Research), the PCR assay was run with all ESBL-producing clinical isolates, the four positive controls, E. coli NCTC 10418 as a non-ESBL-producing control and water as a nontemplate control. Each $20 \mu \mathrm{l}$ reaction tube contained $5 \mu \mathrm{l}$ DNA

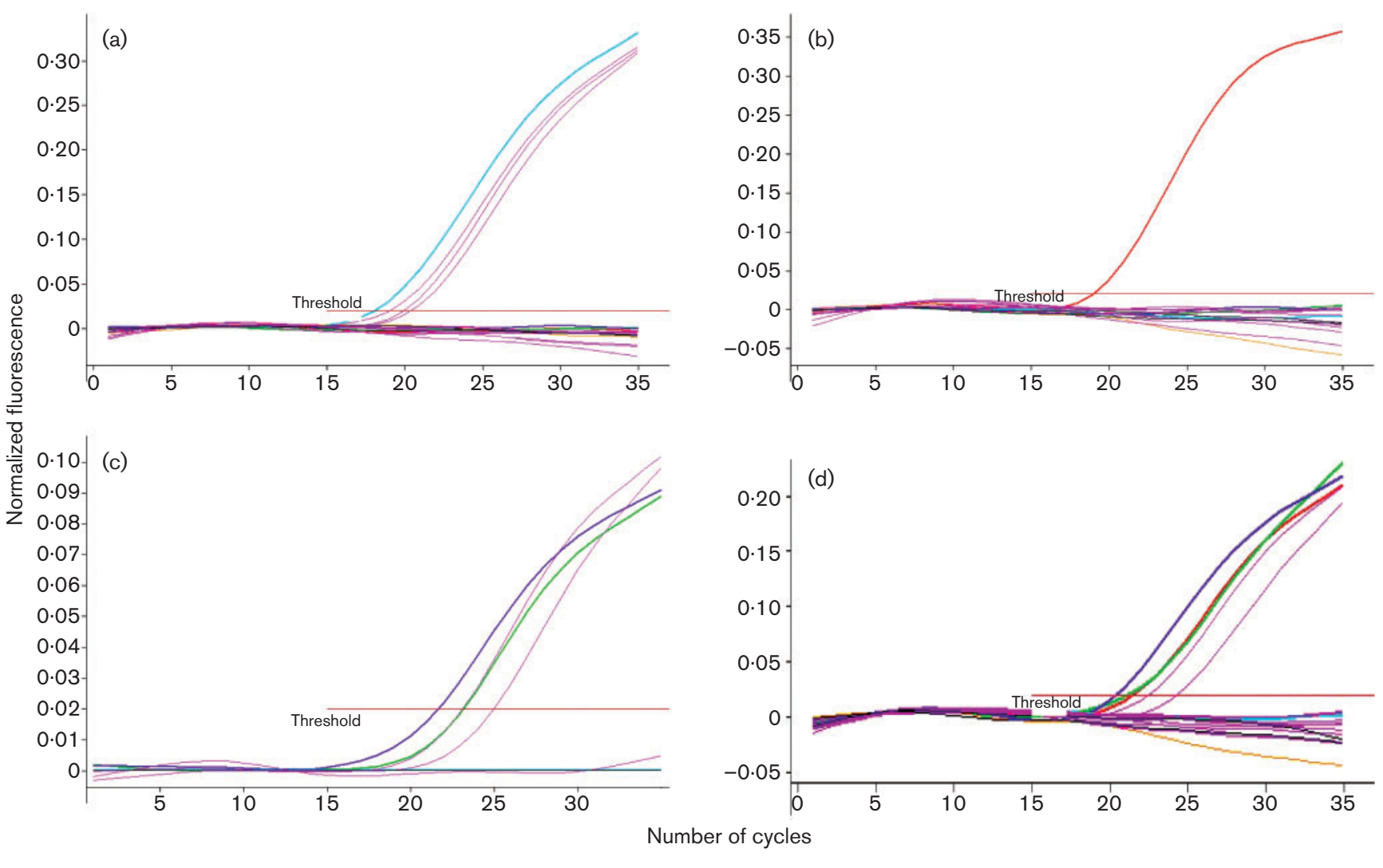

Fig. 1. Single-tube, real-time detection and typing of genes encoding CTX-M ESBLs in 11 blood culture isolates using all four channels of the Rotor-Gene 3000. Negative results show curves below the marked threshold. (a) Joe channel: CTX-M-1 control (bold blue), three positive isolates (purple). (b) Cy5 channel: CTX-M-2 control (bold red), no positive isolates. (c) Rox channel: CTX-M-9 control (bold mauve), CTX-M-14 control (bold green), two positive isolates (purple). (d) Fam channel: positive controls CTX-M-2 (bold red), CTX-M-9 (bold mauve) and CTX-M-14 (bold green), two positive isolates (purple). 
Table 2. ESBL-positive isolates screened by PCR and their assigned genotypes

NT, Not tested.

\begin{tabular}{|c|c|c|c|c|c|c|c|}
\hline Isolate no. & Organism & $\begin{array}{c}\text { Cefotaxime } \\
\operatorname{MIC}\left(\mathrm{mg} \mathrm{l}^{-1}\right)\end{array}$ & $\begin{array}{l}\text { Ceftazidime } \\
\operatorname{MIC}\left(\mathrm{mg} \mathrm{l}^{-1}\right)\end{array}$ & $\begin{array}{c}b l a_{\mathrm{CTX}-\mathrm{M}} \\
\text { detection using } \\
\text { TaqMan assay }\end{array}$ & $\begin{array}{l}\text { CTX-M group } \\
\text { assigned by } \\
\text { TaqMan assay }\end{array}$ & $\begin{array}{l}\text { CTX-M group } \\
\text { assigned by } \\
\text { block-based } \\
\text { reference assay }\end{array}$ & Genotype \\
\hline \multicolumn{8}{|c|}{ Blood culture } \\
\hline 42048 & Pantoea sp. & 16 & $>256$ & - & - & - & - \\
\hline 42049 & Klebsiella pneumoniae & $>256$ & 8 & + & CTX-M-9 & CTX-M-9 & CTX-M-14 \\
\hline 42050 & Escherichia coli & $>256$ & 64 & + & CTX-M-1 & CTX-M-1 & CTX-M-15 \\
\hline 42051 & Escherichia coli & 4 & 32 & - & - & - & - \\
\hline 42052 & Escherichia coli & 16 & 0.5 & + & CTX-M-9 & CTX-M-9 & CTX-M-9 \\
\hline 42053 & Escherichia coli & $>256$ & 64 & + & CTX-M-1 & CTX-M-1 & CTX-M-15 \\
\hline 42054 & Pantoea sp. & 8 & 32 & - & - & - & - \\
\hline 42055 & Klebsiella pneumoniae & 64 & $>256$ & - & - & - & - \\
\hline 42056 & Enterobacter cloacae & $>256$ & $>256$ & - & - & - & - \\
\hline 42057 & Escherichia coli & 128 & $>256$ & - & - & - & - \\
\hline 42058 & Klebsiella pneumoniae & $>256$ & 128 & + & CTX-M-1 & CTX-M-1 & CTX-M-15 \\
\hline \multicolumn{8}{|c|}{ Urine culture } \\
\hline 195009 & Klebsiella sp. & $>256$ & $>256$ & + & CTX-M-1 & NT & NT \\
\hline 195013 & Klebsiella oxytoca & $>256$ & 128 & + & CTX-M-1 & NT & NT \\
\hline 195020 & Klebsiella sp. & $>256$ & 128 & + & CTX-M-1 & NT & NT \\
\hline 195022 & Escherichia coli & $>256$ & $>256$ & + & CTX-M-1 & NT & NT \\
\hline 195026 & Klebsiella ornithinolytica & $>256$ & $>256$ & + & CTX-M-1 & NT & NT \\
\hline 195027 & Escherichia coli & $>256$ & $>256$ & + & CTX-M-1 & NT & NT \\
\hline 195057 & Klebsiella sp. & $>256$ & 32 & + & CTX-M-1 & NT & NT \\
\hline 197015 & Klebsiella pneumoniae & 64 & 128 & - & - & NT & NT \\
\hline 197040 & Pantoea sp. & $>256$ & $>256$ & + & CTX-M-1 & NT & NT \\
\hline 197043 & Escherichia coli & $>256$ & 128 & + & CTX-M-1 & NT & NT \\
\hline 197069 & Klebsiella terrigena & $>256$ & $>256$ & + & CTX-M-1 & NT & NT \\
\hline 197074 & Klebsiella terrigena & $>256$ & 128 & + & CTX-M-1 & NT & NT \\
\hline 197075 & Klebsiella terrigena & $>256$ & $>256$ & + & CTX-M-1 & NT & NT \\
\hline 197076 & Klebsiella terrigena & $>256$ & $>256$ & + & CTX-M-1 & NT & NT \\
\hline 199008 & Klebsiella terrigena & $>256$ & 128 & + & CTX-M-1 & NT & NT \\
\hline 199009 & Klebsiella terrigena & $>256$ & 64 & + & CTX-M-1 & NT & NT \\
\hline 199089 & Klebsiella terrigena & $>256$ & 128 & + & CTX-M-1 & NT & NT \\
\hline
\end{tabular}

extract solution, $2 \mu$ LightCycler FastStart Hybridization kit mixture (Roche Diagnostics), $0.8 \mu \mathrm{l} 25 \mathrm{mM} \mathrm{MgCl}_{2}$ (to give $2 \mathrm{mM}$ magnesium), $0.5 \mu \mathrm{l}$ of each $20 \mathrm{pmol}^{-1}$ forward and reverse primers, $0.2 \mu \mathrm{l}$ of each of the four $10 \mathrm{pmol} \mu^{-1}$ TaqMan probes and $11 \mu \mathrm{l}$ RNase-free water. Cycling conditions were: $10 \mathrm{~min}$ incubation period at $95^{\circ} \mathrm{C}$ followed by 35 cycles of PCR, each cycle consisting of $8 \mathrm{~s}$ at $95^{\circ} \mathrm{C}$ and $60 \mathrm{~s}$ at $58^{\circ} \mathrm{C}$ with a single fluorescence reading for all four channels being taken at the end of the extension stage. Real-time data were analysed with Rotor-Gene software (version 6.0). Upon completion of the run, a cycle threshold (Ct) was calculated by determining the point at which the fluorescence exceeded a threshold limit. This was manually set each time but reproducibly resulted in a normalized fluorescence ranging between 0.01 and 0.02 for all four channels. Any sample demonstrating a fluorescence signal above this value was regarded as positive.

To confirm the specificity of the new assay, the 11 ESBL-producing blood culture isolates were sent to ARMRL for analysis using the reference laboratory standard method, a block-based multiplex PCR (Woodford et al., 2006). The ESBL-producing urine culture isolates were not referred to the ARMRL.
Amplicon sequencing of the PCR-positive blood culture isolates. Real-time PCR products were purified with the QIAquick PCR purification kit (QIAGEN) and their sizes (approx. $300 \mathrm{bp}$ ) were confirmed by agarose gel electrophoresis prior to sequencing

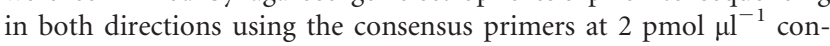
centrations (CEQ 8000 Genetic Analysis System; Beckman Coulter). The amplicon sequences of the four positive controls and all PCR-positive blood culture isolates were compared with published sequences of CTX-M genotypes using the BLAST program (http:// www.ncbi.nlm.nih.gov/BLAST).

\section{RESULTS AND DISCUSSION}

The combination disc method indicated ESBL production in 11 of 34 blood culture isolates resistant to cefotaxime or ceftazidime. The TaqMan PCR assay assigned the four CTX$\mathrm{M}$-producing control isolates to their correct phylogenetic groups and detected $b l a_{\text {CTX-M }}$ in five of 11 ESBL-producing isolates (Fig. 1). No PCR products were observed in the 
negative samples. Three of the five PCR-positive clinical isolates were CTX-M-1 group producers (Fig. 1, Joe channel; two E. coli and one Klebsiella pneumoniae) and two were CTX-M-9 group producers (Fig. 1, Rox and Fam channels; one E. coli and one K. pneumoniae). ARMRL assigned identical CTX-M groups to the same five ESBLpositive isolates with their conventional PCR method (Table 2). BLAST search analysis of the amplicon sequences gave $100 \%$ matches for the assigned genotypes of the four positive controls. The three CTX-M-1 group sequences (isolates 42050, 42053 and 42058) were identical and gave a $100 \%$ match with $b a_{\text {CTX-M-15 }}$ (GenBank accession no. AM40707). The two CTX-M-9 group sequences demonstrated one nucleotide difference; isolate 42049 gave a $100 \%$ match with $b l a_{\text {CTX-M-14 }}$ (GenBank accession no. AJ972957) and isolate 42052 gave a $100 \%$ match with $b a_{\text {CTX-M-9 }}$ (GenBank accession no. AJ416345). However, it is only possible to say that genotypes matched reference sequences over the $300 \mathrm{bp}$ amplified region of the $b a_{\text {СтХ-м }}$ gene. Definitive assignment of any CTX-M-positive isolates to the allelic level would require full sequencing of the entire $b l a_{\text {CTX-M }}$ gene. We concluded that the six CTX-M PCRnegative, ESBL-producing isolates, for which the MICs of ceftazidime were higher than those for cefotaxime, were producing a non-CTX-M ESBL. The CTX-M PCR-negative, ESBL-producing Enterobacter cloacae (isolate 42056; Table 2) showed high-level resistance to both cefotaxime and ceftazidime (MICs both $>256 \mathrm{mg} \mathrm{l}^{-1}$ ), suggesting derepressed production of an AmpC $\beta$-lactamase in addition to the ESBL.

The combination disc method indicated ESBL production in 17 of 444 urinary isolates resistant to ampicillin, co-amoxiclav or cefalexin. The TaqMan assay determined that 16 of 17 ESBL-positive isolates were group 1 CTX-M producers, presumably CTX-M-15 genotypes (Table 2). Isolate 197015 was CTX-M PCR-negative and was therefore assumed to be producing a non-CTX-M ESBL. None of the amplicons from the urinary isolates were sequenced.

These studies support the reported observation (Woodford et al., 2004) that CTX-M genotypes are now the most common ESBL type among UK members of the Enterobacteriaceae, with group 1 CTX-M producers (mostly CTX-M-15) predominating.

In conclusion, we have developed a novel real-time PCR method for the rapid detection and genotyping of CTX-Mproducing members of the Enterobacteriaceae. The method is an improvement on previously described techniques and, to our knowledge, this is the first TaqMan method of its type for CTX-M genotypes. This assay could be useful locally for investigating outbreaks and would be suitable for use in regional or national reference facilities. Use of this method at a local level would reduce the workload of reference laboratories (Woodford \& Sundsfjord, 2005). DNA extraction could be simplified or automated and the Rotor-Gene 3000 apparatus combined with the speed of the assay would allow a large throughput of specimens per $8 \mathrm{~h}$ day.
Importantly, the specificity of the probes negates the time-consuming and costly need for sequencing as major genotypes, encoding group 1, 2 and 9 enzymes (Bonnet, 2004; Livermore \& Hawkey, 2005; Walther-Rasmussen \& Hoiby, 2004), are assigned as a function of the assay. By comparison with conventional PCR techniques, this would make a cheaper and faster same-day service feasible.

\section{ACKNOWLEDGEMENTS}

This work was supported by a grant from Wyeth Pharmaceuticals and was presented in part at the 45th ICAAC, Washington DC, December 2005 (abstract C2-772). We thank Mrs E. Walpole for technical assistance.

\section{REFERENCES}

Bauernfeind, A., Grimm, H. \& Schweighart, S. (1990). A new plasmidic cefotaximase in a clinical isolate of Escherichia coli. Infection 37, 851-858.

Bonnet, R. (2004). Growing group of extended-spectrum betalactamases: the CTX-M enzymes. Antimicrob Agents Chemother 48, $1-14$.

Carter, M. W., Oakton, K. J., Warner, M. \& Livermore, D. M. (2000). Detection of extended-spectrum beta-lactamases in klebsiellae with the Oxoid combination disk method. J Clin Microbiol 38, 4228-4232.

Livermore, D. M. \& Hawkey, P. M. (2005). CTX-M: changing the face of ESBLs in the UK. J Antimicrob Chemother 56, 451-454.

Narayanan, S. K., Nagaraja, T. G., Chengappa, M. M. \& Stewart, G. C. (2001). Cloning, sequencing and expression of the leukotoxin gene from Fusobacterium necrophorum. Infect Immun 69, 5447-5455.

Paterson, D. L. \& Bonomo, R. A. (2005). Extended-spectrum $\beta$ lactamases: a clinical update. Clin Microbiol Rev 18, 657-686.

Paterson, D. L., Ko, W. C., Von Gottberg, A., Casellas, J. M., Mulazimoglu, L., Klugman, K. P., Bonomo, R. A., Rice, L. B., McCormack, J. G. \& Yu, V. L. (2001). Outcome of cephalosporin treatment for serious infections due to apparently susceptible organisms producing extended-spectrum beta-lactamases: implications for the clinical microbiology laboratory. J Clin Microbiol 39, 2206-2212.

Paterson, D. L., Ko, W. C., Von Gottberg, A., Mohapatra, S., Casellas, J. M., Goossens, H., Mulazimoglu, L., Trenholme, G., Klugman, K. P. \& other authors (2004). International prospective study of Klebsiella pneumoniae bacteraemia: implications of extended-spectrum beta-lactamase production in nosocomial infections. Ann Intern Med 140, 26-32.

Walther-Rasmussen, J. \& Hoiby, N. (2004). Cefotaximases (CTX-Mases), an expanding family of extended-spectrum $\beta$-lactamases. Can J Microbiol 50, 137-165.

Woodford, N. \& Sundsfjord, A. (2005). Molecular detection of antibiotic resistance: when and where? J Antimicrob Chemother 56, 259-261.

Woodford, N., Ward, M. E., Kaufmann, M. E., Turton, J., Fagan, E. J., James, D., Johnson, A. P., Pike, R., Warner, M. \& other authors (2004). Community and hospital spread of Escherichia coli producing CTX-M extended-spectrum beta-lactamases in the UK. J Antimicrob Chemother 54, 735-743.

Woodford, N., Fagan, E. J. \& Ellington, M. J. (2006). Multiplex PCR for rapid detection of genes encoding CTX-M extended-spectrum $\beta$ lactamases. J Antimicrob Chemother 57, 154-155. 